\title{
Identifying Misconceptions that Limit Student Understanding of Molecular Orbital Diagrams
}

\author{
Judith L. Jenkins*, Bradley T. Shoopman \\ Department of Chemistry, Eastern Kentucky University, Richmond, Kentucky, United States of America
}

*Corresponding Author: judy.jenkins@eku.edu

\section{ABSTRACT}

Molecular orbital diagrams are commonly taught and used for describing chemical bonding chemistry courses including courses at the college level. Misconceptions that occur when students construct and/or use these diagrams are difficult to anticipate, diminish the diagram utility, and limit student learning. In this exploratory study, a combination of think-aloud activities and written probes was used to identify misconceptions that persist into upper-level college chemistry courses. While the majority of students could properly construct molecular orbital diagrams, many struggled to use and interpret the resulting diagrams. Pronounced disconnects between the depiction of chemical bonds and how these depictions demonstrate molecular properties such as stability and reactivity were observed, informing strategic curricular interventions.

KEY WORDS: chemistry; chemical bonding; constructivism; misconceptions

\section{INTRODUCTION}

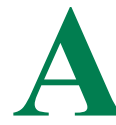
misconception is an idea, notion, or thought that does not mirror reality nor is it grounded in scientific reasoning (Luxford and Bretz, 2014; Nakhleh, 1992). It is important to identify, examine, and strategically address misconceptions held by students so that they progress from descriptions and definitions to richly interconnected, functional understanding. The key goal of this exploratory work is the elucidation of misconceptions encountered by college-level chemistry students learning and using molecular orbital theory to depict chemical bonding. Substantial effort has been invested in identifying and reconciling misconceptions at the high school and $1^{\text {st }}$ year college levels (see for instance Birk and Kurtz, 1999; Luxford and Bretz, 2014; and Papageorgiou and Zarkadis, 2016). Here, we build on this work by focusing on college chemistry majors within 1 year of completing their undergraduate degrees. These students have been exposed to wide-ranging chemical concepts by a variety of instructors. Before detailing the results of our study, we present an overview of the previous research efforts into misconceptions in chemistry with a particular focus on misconceptions related to models of chemical bonding.

\section{LITERATURE REVIEW}

Misconceptions in chemistry are well documented (Nakhleh, 1992; Peterson and Treagust, 1989). The abstract nature of this discipline requires students to understand that matter and the changes it undergoes are the result of interactions between difficult to observe and often indistinguishable particles (i.e., electrons, atoms, and molecules). Educators use models that are specifically useful for expressing a particular molecular-level property or event but may be broadly incomplete. As a result, non-scientific mental models can arise when students struggle to connect a representation to the corresponding chemical phenomenon or when they do not understand the limitations of a particular model (Papageorgiou and Zarkadis, 2016; Halim et al., 2013). In addition, the density of information in chemical representations may be only partially grasped by newer learners. For example, Kelly et al. (2010) demonstrated that while students could depict chemical reactions that clearly showed atomic rearrangement, they struggled to predict whether a chemical reaction would give rise to any observable macroscopic change to the contents of a beaker. Strategic remediation requires that student misconceptions must first be observed and understood as evidenced by extensive research efforts in this area to date (see for instance Papageorgiou and Zarkadis, 2016; Luxford and Bretz, 2014; Birk and Kurtz, 1999) and motivating the current work.

\section{Misunderstanding Chemical Bonds}

Understanding chemical bonding is critically important for chemists, but the complexity of bonds complicates teaching and learning efforts (Özmen, 2004). Many students view bonds as entities linking atoms together, rather than the movement and attraction of electrons (Halim et al., 2013; Kelly et al., 2010). Moreover, teaching ionic versus covalent bonding models leads to the persistent belief that only two distinct types of bonds exist and that they are opposite from one another (Robinson, 1998). Similarly, students view the octet "rule" as the cause for reactions rather than using it as a guide to identify stable molecules (Robinson, 1998). General bonding 
misconceptions include drawing covalent bonds between ions, not understanding when disassociation occurs, assuming that aqueous reactants are molecular pairs before reacting, and believing that reactant molecules break apart on mixing (Halim et al., 2013; Kelly et al., 2010).

Of particular interest ways, representations of chemical bonds may contribute to misconceptions because these contributions, when identified, can be strategically addressed by educators. For instance, a Lewis structure is one commonly used, wellstudied way of representing a molecule that explicitly depicts chemical bonds. Beginning in the $1^{\text {st }}$ year college chemistry (or earlier), students learn to construct Lewis structures by considering interactions between valence electrons of the constituent atoms. When constructed accurately, these structures contain a wealth of information relevant to a molecule's properties in a concise manner. However, flaws and ambiguities in students' abilities to produce valid structures will prevent the development of representational competence with Lewis structures (Luxford and Bretz, 2014; Cooper et al., 2010). After analyzing responses from 176 students, Cooper et al. (2010) noted that 30-40\% of students believe molecular shape, chemical properties, or physical properties cannot be predicted from Lewis structures even though structural information, combined with other models, allow us to use Lewis structures to estimate these properties.

\section{Misunderstanding Chemical Interactions}

Understanding non-covalent interactions and intermolecular forces allow students to predict and explain observable phenomena including phase changes and solubility. Students confuse non-covalent and covalent interactions, often misunderstanding intermolecular forces such as hydrogen bonding to be interactions between atoms in a bond, rather than interactions between molecules (Cooper et al., 2015; Cooper et al., 2010; Nakhleh, 1992; Peterson and Treagust, 1989). For instance, after instructors stated that water has a high boiling point due to the strong hydrogen bonds that must be overcome, many students begin to believe covalent bonds are being broken during a phase change (Cooper et al., 2015). Anthropomorphic attributions are often used to explain the behaviors of atoms and molecules, but this common practice has been shown to create the misconception that matter is alive (Nakhleh, 1992). As a result, students believe that the movement of atoms, molecules, and ions is directed by some force of will and that matter just "knows" where to go, rather than the random process that more accurately describes chemical reactions (Kelly et al., 2010). Similarly, canceling out spectator ions to obtain the net ionic equation causes students to think that matter is being canceled out or disappearing (Kelly et al., 2010). Other related misconceptions identified by researchers include the inability to depict phase changes in drawings (Cooper et al., 2013), believing that molecules have different weights in different phases, thinking that molecules change shape between phases (Nakhleh, 1992), and assuming that individual atoms are liquids, solids, or gasses depending on state (Papageorgiou and Zarkadis, 2016).
All models, concepts, and topics in chemistry build on each other in a cumulative fashion, so misconceptions at any level are detrimental to students' future learning endeavors - especially in more advanced courses. Often, a single misconception is not what dooms students, but rather a "set of loosely connected ideas, skills, and heuristics that are not well integrated" (Cooper et al., 2013. p. 699). To facilitate opportunities for students to construct meaningful, integrated chemical understanding, we must strategically confront student misconceptions. While some misconceptions may seem obvious in retrospect, it is important to observe and to correct students as they construct meaning so that misconceptions do not go unresolved, motivating the efforts described here.

Using a combination of think aloud and written probes, we examined ways students use molecular orbital theory to explain and predict chemical bonding. Specific research questions include (i) what are students thinking when construction molecular orbital diagrams and (ii) what are students thinking when using these diagrams to determine the relative stabilities of similar molecules. While the majority of students could properly construct molecular orbital diagrams, many struggled to use and interpret the resulting diagrams. Pronounced disconnects between the depiction of chemical bonds and how these depictions demonstrate molecular properties such as stability and reactivity were observed. These findings inform future curricular design.

\section{METHODOLOGY}

\section{Research with Human Subjects}

The authors completed all required training for human subject research, offered through Eastern Kentucky University's (EKU, 2018) online subscription to the Collaborative Institutional Training Initiative (EKU IRB, 2018). The study was approved through EKU's Institutional Review Board (EKU Office of Institutional Research, 2017) in the expedited category. EKU students were made aware of the study through both written and verbal explanations; participation, in the study, was optional and did not impact course grades. While all students completed the activities, the results presented here only contain response from students providing their written consent.

\section{Description of the School and Students}

Eastern Kentucky University, a regional comprehensive university located in Richmond, Kentucky, USA, serves approximately 14,000 undergraduate students and 2500 graduate students (EKU Office of Institutional Research, 2017). As a school of opportunity, 53\% of EKU Spring 2018 graduates were low income and $47 \%$ were the first-generation college students (EKU, 2018). This study was conducted in an upper-level inorganic chemistry course during the fall 2016 semester (13 students). The course met twice weekly for $75 \mathrm{~min}$ throughout the 16-week semester. Students enrolled in this course were chemistry majors within 1 year of graduating; course prerequisites include two semesters of general chemistry and two semesters of organic chemistry. 
Molecular orbital theory is not introduced in EKU's general chemistry courses, which students complete in their $1^{\text {st }}$ year. In their $2^{\text {nd }}$ year, students do encounter molecular orbital theory in organic chemistry. Students recognize constructive overlap of atomic orbitals such that electron density is distributed across multiple atomic nuclei, yielding sigma and pi bonds in small hydrocarbons, and there is some introduction to pi-pi* transitions. Although students encountered models of chemical bonding throughout the chemistry curriculum, there is minimal exposure to advanced bonding models before inorganic chemistry.

Student learning outcomes in the course hosting this study were informed by students' exposure to chemical bonding models in the prerequisite courses and are summarized here. Students completing inorganic chemistry should be able to use their understanding of chemical bonding models to explain chemical bonds and to predict properties (including physical properties, stability, reactivity, and functionality) of inorganic compounds. Bonding models included in this course are molecular orbital theory, group theory, crystal field theory, and ligand field theory, but discussion of course content and objectives is limited to molecular orbital theory for this manuscript. Students provide evidence that they understand and can apply molecular orbital theory to describe chemical bonding when they (i) sketch shapes of bonding, non-bonding, and antibonding molecular orbitals, (ii) construct molecular orbital diagrams for homonuclear and heteronuclear small molecules, applying group theory as needed to determine orbitals contributing to the bonds, and (iii) determine relative bond lengths and bond strengths from molecular orbital diagrams; predict lowest energy bond formation.

\section{Study Details}

This exploratory study consisted of three phases. The first phase, think aloud, was completed in the $2^{\text {nd }}$ week of the semester. Following a brief review of the molecular orbital theory concepts encountered in the prerequisite courses, students met one-on-one with the instructor for the activity. Each student was given the same problems to solve, and the student was asked to speak their thoughts aloud while writing out solutions. Students' verbal responses were recorded, transcribed, and analyzed alongside the written responses. The second phase, interventions, consisted of targeted activities designed to address some of the misconceptions revealed during the think-aloud phase. These interventions were implemented alongside other course contents and are described in the following section for context. The third phase, written probe, consisted of problems from the course final examination. Students' written responses were analyzed for further evidence of misconception, both those that may have persisted throughout the semester and those newly arisen. We hypothesize that results gathered through these means will elucidate student misconceptions and inform strategies for more integrated learning strategies.

We recognize the limits of this study, namely, the small sample size and the purely qualitative analysis of student responses.
Nevertheless, the data presented here can be compared to the existing literature to help determine whether or not such misconceptions are actually specific to molecular orbital theory or additional examples of well-documented points of confusion among chemistry learners. In addition, these findings can inform both targeted interventions and future, more comprehensive studies. Ultimately, this work demonstrates manageable, meaningful strategies for revealing students' thought processes as they encounter complex content.

\section{RESULTS AND DISCUSSION}

\section{Think Aloud}

During the think-aloud activity, students attempted to (1) generate molecular orbital diagrams for $\mathrm{O}_{2}, \mathrm{O}_{2}^{-}$, and $\mathrm{O}_{2}^{+}$and (2) rank these species of increasing stability. While writing out their responses, students spoke their thoughts aloud describing their actions, explaining choices, and articulating questions. Then, students completed a metacognitive probe assessing their confidence in their answers. For each answer, students could rank themselves as highly confident, somewhat confident, neither sure nor unsure, somewhat unsure, or highly unsure. While students were completing the activity, the instructor verbally prompted students to speak their thoughts but did not provide input. After the activity was completed, the instructor worked with the student to affirm correct work and address questions and/or misconceptions.

Nine of the 13 students correctly constructed molecular orbital diagrams for the diatomic oxygen species, yielding drawings similar to Figure 1a; all of these students described themselves as highly or somewhat confident. Two students were able to determine the electron configuration for an oxygen atom but did not use this information to construct molecular orbital diagrams. Instead of using six valence electrons as shown in Figure 1a, these students used eight valence electrons per oxygen atom, justifying this choice with the octet rule. One student used the Bohr model as shown in Figure 1b and one student simply could not remember how to approach the problem. Five students correctly determined the relative

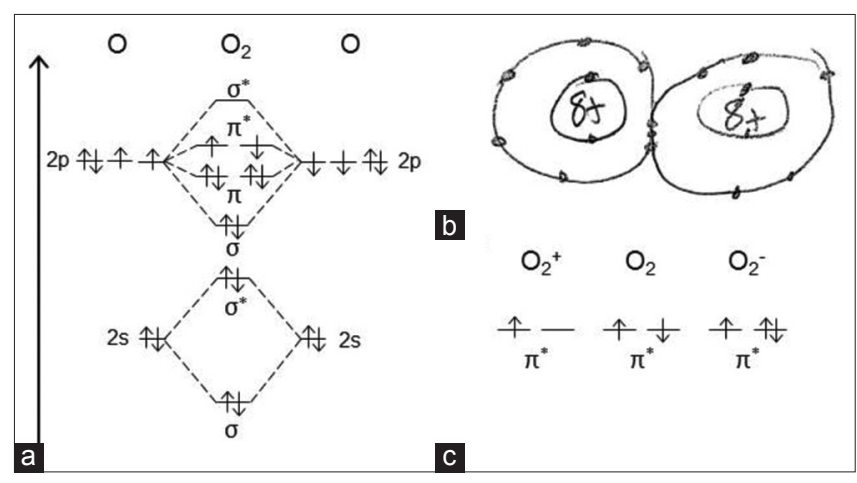

Figure 1: Responses to the think-aloud questions include an accurate, qualitative molecular orbital diagram of oxygen (a), an incorrect student response invoking the Bohr model (b), and comparison of the number of electrons in antibonding orbitals for the three oxygen species (c) 
stability of the diatomic oxygen species $\left(\mathrm{O}_{2}^{+}>\mathrm{O}_{2}>\mathrm{O}_{2}^{-}\right)$by calculating bond order and/or by comparing the number of electrons in the antibonding $\pi^{*}$ orbitals (Figure 1c). All five students were somewhat or highly confident of their answers. Of the eight students answering incorrectly, four expressed some confidence in their incorrect answers and four were either neutral or unsure of their answers.

Students answering incorrectly articulated numerous misconceptions. Some thought the increased number of electrons in $\mathrm{O}_{2}^{-}$relative to $\mathrm{O}_{2}$ made the anion most stable because "all species want to be noble gases." Others thought that the anion would be the most stable because "oxygen is electronegative and therefore wants more electrons." The most consistently articulated incorrect rationale was that neutral $\mathrm{O}_{2}$ is most stable because it is "prevalent in nature" and/or because "neutrals less reactive than ions." It is not difficult to see how students arrived at these mistaken conclusions, invoking somewhat accurate but not necessarily relevant nor accurately applied pieces of chemical information encountered in chemistry courses. Nevertheless, it is clear that despite knowing how to construct an accurate molecular orbital diagram, many students did not understand some of the chemical information communicated through molecular orbital diagrams. In general, these misconceptions are consisted with those documented by others, particularly with respect to the octet rule (Robinson, 1998).

Based on these misconceptions, we suggest that activities teaching molecular orbital theory be accompanied by explicit, intentional decoding of molecular orbital diagrams. In addition to teaching how molecular orbital diagrams are constructed, students should be challenged to describe how these diagrams are interpreted and applied. Preliminary strategies for scaffolding and enhanced conceptual understanding are given in the following section.

It is interesting to compare students' confidence to the accuracy of their responses. For the task requiring lower-order thinking skills (remember), student perceptions matched their answers. Students providing correct answers were somewhat or highly confident, while most of those answering incorrectly expressed uncertainty. When higher-order thinking skills were needed (compare, analyze, and judge), some students expressed strong confidence in their wrong answers. Metacognitive probes similar to the one used here have been shown to help calibrate student's perceptions of their comprehension (Cook et al., 2013). We tentatively suggest that metacognitive discrepancies may be used to help pinpoint areas where misconceptions persist. When students display a mismatch between perceived and actual comprehension, they are selfidentifying limiting knowledge gaps that may, in some cases, result from misunderstandings.

\section{Interventions}

Multiple interventions were implemented between the preliminary think-aloud exercise and the written probes. These interventions were designed to highlight different pieces of chemical information represented by molecular orbital diagrams; two examples are discussed here for context. First, during an in-class activity, students were shown the diagrams in Figure 2 and asked to identify the diagram corresponding to a homonuclear diatomic species. To justify their answers, students compared the relative energies of the atomic orbitals, reinforcing the use of experimental data (potential energies of electrons in atomic orbitals) to construct the molecular orbital diagrams. More broadly, the whole-class discussion accompanying this intervention emphasized that, rather than arbitrary choices, the energetic ordering of orbitals communicates meaning about the identity of the species involved and the potential energy of the electrons in both the contributing atoms and the resulting molecule.

On an open-resource homework assignment, students used a molecular orbital diagram of carbon monoxide (CO) to describe orbital interactions in a metal carbonyl bond. First, students identified the $\mathrm{CO}$ molecular orbital donating electron density to the metal ion in a sigma-type fashion. The correct orbital must be occupied, appropriately shaped to form a sigma-type interaction, and sufficiently reactive (high in potential energy). Then, students identified the $\mathrm{CO}$ molecular orbital that could accept electron density from a metal ion in a pi-type interaction. The correct orbital must be unoccupied, appropriately shaped to form a pi-type interaction, and sufficiently reactive (high in potential energy). Next students were asked to discuss the impacts of the metal carbonyl bond on the carbonyl bond strength, predicting how the CO IR stretching frequencies for free and bound $\mathrm{CO}$ would differ. The sigma-type electron donation decreases the electron density in a bonding $\mathrm{CO}$ molecular orbital, and the pi-type electron acceptance increases the electron density in an antibonding $\mathrm{CO}$ molecular orbital, both of which ultimately decrease the $\mathrm{CO}$ bond strength and, therefore, decrease the vibrational frequency. This activity guided students as they connected the components of a molecular orbital diagram (occupied and unoccupied orbitals, orbital shapes, and orbital energies) to the utility of the diagram in describing/predicting further bonding interactions. Then, these connections were extended to experimentally observable phenomena (IR spectra of metal carbonyl complexes).

\section{Written Probes}

Questions on the course final examination were used as written probes to identify new and/or persisting misconceptions. After constructing a molecular orbital diagram for NO, students answered the following questions: How does your diagram

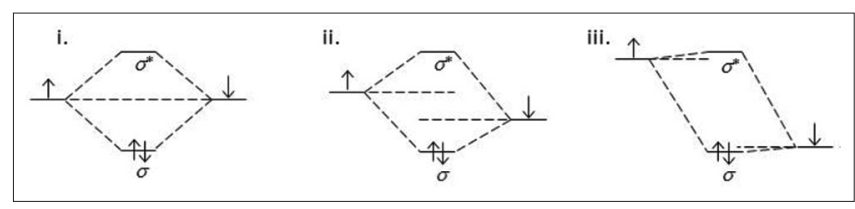

Figure 2: Sample in-class activity. Which of the generic molecular orbital diagrams above could correspond to a homonuclear diatomic molecule? What evidence supports your answer(s)? 
demonstrate the differences in electronegativity for oxygen and nitrogen? Of the three species $\mathrm{NO}, \mathrm{NO}^{+}$, and $\mathrm{NO}^{-}$, which would you predict to have the shortest bond? Explain. A periodic table was the only available resource for students while answering these questions.

Eleven of 13 students correctly identified ways, in which the molecular orbital diagram for NO illustrated the difference in electronegativity between oxygen and nitrogen. These students noted that the oxygen atomic orbitals appear at lower potential energies than those of nitrogen and that the molecular orbitals are closer in potential energy to the oxygen atomic orbitals rather than the nitrogen atomic orbitals. Students answering incorrectly used the fact that oxygen "contributes more electrons" as evidence of relative differences in electronegativity. Ten of 13 students correctly determined the relative bond lengths of the diatomic $\mathrm{NO}$ species $\left(\mathrm{NO}^{-}>\mathrm{NO}\right.$ $>\mathrm{NO}^{+}$) using bond order and/or relative number of electrons in the highest energy antibonding molecular orbitals to justify their answers. Students answering incorrectly misinterpreted the implications of bond order, thinking that the lowest bond order corresponded to the shortest bond. The measurable improvement in student's understandings of molecular orbital diagrams indicates that the described interventions did facilitate deeper, more meaningful comprehension of these bonding representations. The misconceptions persisting show ways, in which students misapply formulaic procedures (such as calculating bond order) that can be memorized but may not be fully understood.

\section{CONCLUSIONS}

The misconceptions identified here inform future strategies for teaching molecular orbital theory. Before targeted interventions, students used familiar concepts to explain the less familiar. To determine relative stability of diatomic oxygen species, many relied on personal experience (neutral $\mathrm{O}_{2}$ is prevalent in nature) or misapplied "rules" (such as the octet rule). Their confidence in these inaccurate justifications showed that students were unaware of their own misconceptions. After interventions, the majority of students adopted chemically reasonable strategies, though some used memorized formulaic approaches (bond order) incorrectly, evidence of persisting confusion.

These misconceptions are specific to a particular bonding model, but more broadly, they are additional examples of the ways, students struggle to see the interconnectedness of chemical concepts across the curriculum. Proposed solutions to this challenge range from lesson-specific interventions similar to those discussed here to more holistic curricular overhaul. Specific interventions are easily implemented by individual instructors without requiring extensive restructuring of course content and delivery. Such solutions are also helpful where multiple instructors teach sections of the same course with some level of autonomy, where lecture and laboratory content need to remain on a similar schedule, etc. Comprehensive reorganization and reframing of chemical content such that the interconnectedness of chemical concepts is explicit requires more holistic overhaul but may also result in fewer limiting disconnects. Efforts to this end at the P-12 level are evident in the Next Generation Science Standards, a three-dimensional approach to science education shaped around what students should know (Disciplinary Core Ideas), how concepts are related (Crosscutting Concepts), and necessary skills (Science and Engineering Practices) (National Research Council, 2012). Instructional strategies such as Modeling Instruction are practical examples of NGSS-aligned holistic approaches (Cullen, 2015; Dukerich, 2015). At the college level, an approach like chemical thinking (Sevian and Talanquer, 2014) introduces to students to chemistry as a way of thinking rather than a body of content.

Unexpected benefits of this study were seen in students' behavior and in their written evaluations of the course. Following the think-aloud exercises, students' willingness to ask questions and discuss problems in class increased noticeably, as if the students gained some level of confidence in their abilities to figure things out (and admit what they do not know). Participation in out-of-class, optional office hours also increased; students seemed less intimidated to seek out help. Perhaps most surprisingly, by letting the students know that they were part of a research study, students were able to see themselves through the instructor's eyes. One student noted that the instructor "actually realizes that learning is hard for us sometimes." Another commented that the instructor "didn't just want us to get the right answer. (The instructor) wanted us to understand why our answers were right." While these outcomes were not anticipated, they suggest that instructor transparency can positive impact students.

This work contributes to our awareness of misconceptions that persist through college chemistry. Not surprisingly, students return to familiar though not necessarily applicable explanations when faced with new challenges. However, revealing these misapplications can be readily accomplished using simple think-aloud activities - extending the impact of this work beyond the specifics of molecular orbital diagrams. Ultimately, deep student learning requires continued vigilant attention to student misconceptions and informed strategic interventions.

\section{ACKNOWLEDGMENTS}

The authors gratefully acknowledge contributions of Eastern Kentucky University students in the fall 2016 CHE 450 cohort as well as Angel Spurlock, Shanlee Burton, and Jessica Nelson in preliminary data collection and analysis.

\section{REFERENCES}

Birk, J.P., \& Kurtz, M.J. (1999). Effect of experience on retention and elimination of misconceptions about molecular structure and bonding. Journal of Chemical Education, 76(1), 124.

Cook, E., Kennedy, E., \& McGuire, S.Y. (2013). Effect of teaching metacognitive learning strategies on performance in general chemistry courses. Journal of Chemical Education, 90(8), 961-967. 
Cooper, M.M., Corley, L.M., \& Underwood, S.M. (2013). An investigation of college chemistry students' understanding of structure property relationships. Journal of Research in Science Teaching, 50(6), 699-721.

Cooper, M.M., Grove, N., Underwood, S.M., \& Klymkowsky, M.W. (2010). Lost in Lewis structures: An investigation of student difficulties in developing representational competence. Journal of Chemical Education, 87(8), 869-874.

Cooper, M.M., Williams, L.C., \& Underwood, S.M. (2015). Student understanding of intermolecular forces: A multimodal study. Journal of Chemical Education, 92(8), 1288-1298.

Cullen, D.M. (2015). Modeling instruction: A learning progression that makes high school chemistry more coherent to students. Journal of Chemical Education, 92(8), 1269-1272.

Dukerich, L. (2015). Applying modeling instruction to high school chemistry to improve students' conceptual understanding. Journal of Chemical Education, 92(8), 1315-1319.

Eastern Kentucky University (EKU). (2018). Spring 2018 Commencement. Available from: https://www.ir.eku.edu/insidelook/spring-2018commencement. [Last retrieved on 2018 Dec 5].

Eastern Kentucky University Institutional Review Board (EKU IRB). (2018). Institutional Review Board. Available from: http://www. sponsoredprograms.eku.edu/institutional-review-board. [Last retrieved on 2018 Apr 5].

Eastern Kentucky University Office of Institutional Research (EKU Office of Institutional Research). (2017). Enrollment. Available from: https:// www.ir.eku.edu/factbook-2016-2017. [Last retrieved on 2018 Dec 5].

Halim, N.D.A., Ali, M.B., Yahaya, N., \& Said, M.N.H. (2013). Mental model in learning chemical bonding: A preliminary study. Procedia-Social and
Behavioral Sciences, 97, 224-228.

Kelly, R.M., Barrera, J.H., \& Mohamed, S.C. (2010). An analysis of undergraduate general chemistry students' misconceptions of the submicroscopic level of precipitation reactions. Journal of Chemical Education, 87(1), 113-118.

Luxford, C.J., \& Bretz, S.L. (2014). Development of the bonding representations inventory to identify student misconceptions about covalent and ionic bonding representations. Journal of Chemical Education, 91(3), 312-320.

Nakhleh, M.B. (1992). Why some students don't learn chemistry: Chemical misconceptions. Journal of Chemical Education, 69(3), 191.

National Research Council. (2012). A Framework for K-12 Science Education: Practices, Crosscutting Concepts, and Core Ideas. Washington, DC: The National Academies Press.

Özmen, H. (2004). Some student misconceptions in chemistry: A literature review of chemical bonding. Journal of Science Education and Technology, 13(2), 147-159.

Papageorgiou, G.M.A., \& Zarkadis, N. (2016). Understanding the atom and relevant misconceptions: Students' profiles in relation to three cognitive variables. Science Education International, 27(4), 464-488.

Peterson, R.F., \& Treagust, D.F. (1989). Grade-12 students' misconceptions of covalent bonding and structure. Journal of Chemical Education, $66(6), 459$.

Robinson, W.R. (1998). An alternative framework for chemical bonding. Journal of Chemical Education, 75(9), 1074.

Sevian, H., \& Talanquer, V. (2014). Rethinking chemistry: A learning progression on chemical thinking. Chemistry Education Research and Practice, 15(1), 10-23. 